\title{
Configuration and Coordination issues in a Virtual Enterprise Environment
}

\author{
L. M. Camarinha-Matos, C. Lima \\ New University of Lisbon - Faculty of Sciences and Technology \\ Quinta da Torre - 2825 Monte Caparica - Portugal \\ Phone +351-1-2948517 Fax +351-1-2941253 \\ E-mail \{cam,cpl\}@uninova.pt
}

\begin{abstract}
An architecture to support the implementation of a computer-based infrastructure to allow the participation of Small and Medium size Enterprises (SMEs) in a Virtual Enterprise (VE) environment is presented emphasizing the configuration and coordination aspects. The architecture is employing the new emerging standards and advanced technologies in communication, cooperative information management and distributed decision making. The multi-level coordination approach adopted in the PRODNET architecture ${ }^{1}$ is briefly presented, focusing the hierarchical workflow management aspects. The proposed approach provides the flexibility, privacy and customization support for a company within a VE Environment as well as the internal coordination of all events/messages arriving at the company.
\end{abstract}

\section{Keywords \\ Virtual Enterprise, Workflow systems, Cooperative Systems}

\footnotetext{
${ }^{1}$ This work is based on the ongoing activities of the European Union funded project called PRODNET II, the ESPRIT project 22647. It involves partners from Europe and Latin America, including Research Institutes, engineering companies and end users. (Home page http://www.uninova.pt/ PRODNET.)
} 


\section{INTRODUCTION}

Several on going research projects dealing with Virtual Enterprises (VE) can be found around the world nowadays. Examples are the NIIIP "family" of projects [Niiip96] and CommerceNet in the USA and Canada; the NCALS [Mizuta97] in Japan; SCM+ [Camarinha97a], X-CITTIC, MARVELOUS, VENTO, VEGA [Zarli97], PLENT and PRODNET II [Camarinha97b, Camarinha97c, Afsarmanesh97a, Afsarmanesh97b] in the European Union. This large list only reinforces the point that a big effort is being applied to deeply understand and materialize the paradigm of Virtual Enterprise, which is becoming vital to those enterprises aiming to survive in a more and more competitive "Internet Age".

The natural barriers (physical, geographical, market segment, etc.) to getting different companies in doing business together, are being strongly reduced by the technological advances in the communication technologies and computer networks. The companies (specially the SMEs) must join skills and resources in order to survive and gain competitive advantage in a global market environment. However, a definition of a reference architecture and the design and development of a supporting platform and appropriate protocols and coordination mechanisms are still required to really materialize the VE paradigm [Camarinha97c].

In terms of the coordination models for a VE, there are a large number of factors that impose a flexible approach. Examples of such factors are:

- Diversity of VE classes, in terms of duration, topology, coordination policy, visibility scope, etc.

- Diversity of roles played by each enterprise, such as VE member, coordinator, client and supplier.

- Diversity of internal management policies and socio-organizational structures found in each company.

- Diversity of rights and duties that can be associated to each VE member.

- Diversity of contract / subcontract forms.

- Participation of a company in multiple VEs.

- Evolution of support technologies, safety mechanisms and the legal framework for electronic commerce.

Besides these factors, it shall be noted that forms of interaction and cooperation between enterprises are likely to evolve with experience and trust building mechanisms.

This paper describes the current approach being developed by the Esprit project PRODNET II, which aims at designing and developing an open platform to support industrial virtual enterprises with special focus on the needs of small and medium size enterprises. The emphasis is put on the Configuration and Coordination aspects of the PRODNET Architecture.

PRODNET II assumes that once SMEs realize that using a feasible and reliable computational infrastructure to support their participation in the VE environment (configured according to their particular needs), they might go into a progressively deeper integration of efforts and competencies in order to increase their profits as well as their "arsenal" to fight against the big companies. However this is not a 
one-step process. Therefore, appropriate mechanisms for a gradual increment in the level of cooperation and sharing must be offered by the supporting platform.

A more detailed description about the architecture itself can be found in [Camarinha97b, Camarinha97c, Afsarmanesh97a, Afsarmanesh97b].

\section{PRODNET GENERAL APPROACH}

The PRODNET approach is strongly based on the fact that a company already exists before it decides to participate in a VE environment. Therefore, such company usually has an internal environment (legacy systems), which has to be kept as far as possible independent of any VE environment. The link between both the internal and the VE environment has to be provided in a configurable way, by any computational platform offering support to the participation in a VE environment.

On the other hand, and from the network perspective, a general requirement for an infrastructure to support VEs is that companies must be able to inter-operate and exchange information in real time so that they can work as a single integrated unit although keeping their independence / autonomy. Thus, the PRODNET platform is positioned between two very different worlds: the company internal world and the external (network) world. It is mandatory to preserve the harmony and the wellbalanced interoperation among them.

The PRODNET approach shows a clear separation between the functionalities related with both "worlds", which allows the definition of two main modules within the architecture: the Internal Module and the Cooperation Layer (fig. 1). The Internal Module represents the autonomous unit of a particular company, including the complete structure of the company's information (databases, information systems, etc.) and all the internal decision making processes / enterprise activities (internal PPC and engineering systems). The Cooperation Layer contains all the functionalities for the inter-connection between the company and the VE environment. It represents the communication and coordination role and works as the company's interlocutor within the VE environment.

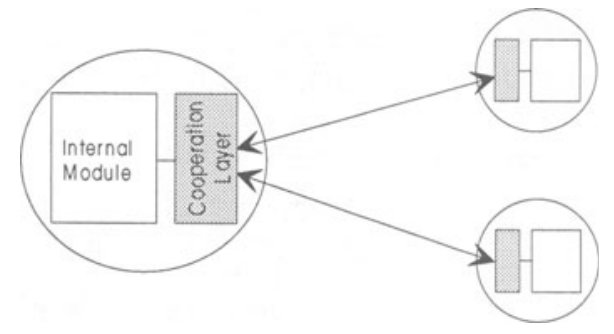

Figure 1 - PRODNET General Approach

A schematic view of the architecture can be seen in figure 2. The description of the set of functionalities considered by PRODNET to support a VE can be found in [Camarinha97a, Camarinha97c].

The main components of the architecture are: 
- PPC - Production Planning and Control System. This is the most important component of the internal module of a VE node, which includes functions such as: Industrial Logistics Management: Orders flow management, Product data management, Sales Forecasts handling, Actual Requirements Planning; Master Production Scheduling; Production Control; Quality Control I Tracking; and Industrial Costing.

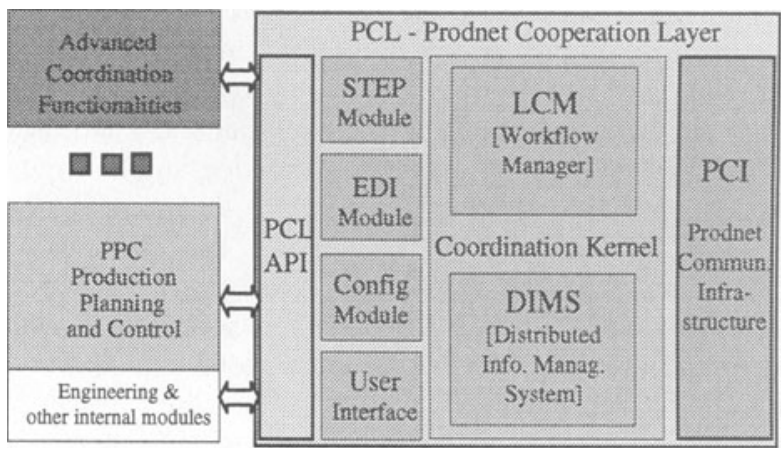

Figure 2 - PRODNET General Architecture

- DIMS - Distributed Information Management System. The Distributed Information Management Subsystem in the PRODNET Cooperation Layer is responsible to model and manage all cooperation support information.

- EDI Module. This module is responsible for receiving and formatting ordersrelated messages in EDIFACT format.

- STEP Module. The STEP module's function is to handle the technical product data used within PRODNET. Ideally all product data should be exchanged in STEP format. The STEP services provided to PRODNET will allow the transmission and reception of STEP files that have been clear text encoded according to a defined schema.

- PCI - PRODNET Communication Infrastructure. This module is responsible for handling all communications with the other nodes in the network assuring basic safety and authentication mechanisms.

- Configuration and User Interface. As mentioned above, the PRODNET platform is intended to support a large diversity of enterprises and interconnection modes, corresponding to a large heterogeneity in terms of available / installed services and desired management procedures. Therefore it is necessary to specify the desired cooperation behavior in an explicit plan that will be "executed"/controlled by the Local Coordination Module (LCM).

- ACF - Advanced Coordination Functionalities are related to the global coordination of the VE and partners search and selection.

From now on the discussion will be focused on the PCL management. 


\section{PCL CONFIGURATION AND MANAGEMENT}

\subsection{General requirements}

The PRODNET platform is intended to support a large diversity of enterprises and interconnection modes, varying from a small company with a networked PC, to a medium or large company with some legacy systems - like a "classical" PPC system. As the PRODNET platform cannot impose tight rules to the companies, it must be configurable in order to support a variety of scenarios. For instance, in order to guarantee that both privacy and autonomy of each company will be preserved within a VE environment, the PCL behavior must be configured according to the needs of each company. Different companies have different internal environments and they make business in different ways. For example, a company might need a strong human-based control of the client orders whilst others may prefer to rely on the PPC system. Therefore, it is really necessary that each company can explicitly configure its desired cooperation behavior. Another configurable issue is the data sharing among VE members. The company itself should be able to explicitly authorize the access rights to its internal data. Finally, each company might have a particular way of handling the services provided by PCL. The Local Configurator Module (LCF) is the component responsible for all configuration issues related to PCL.

As shown previously, PCL is composed of several modules which require a coordination/management function among those modules. The Local Coordinator Module (LCM) is the component responsible for their interoperation. As configuration and coordination are inter-related issues, LCM and LCF have to work in a close liaison.

Another key issue related to both configuration and management is the service concept.

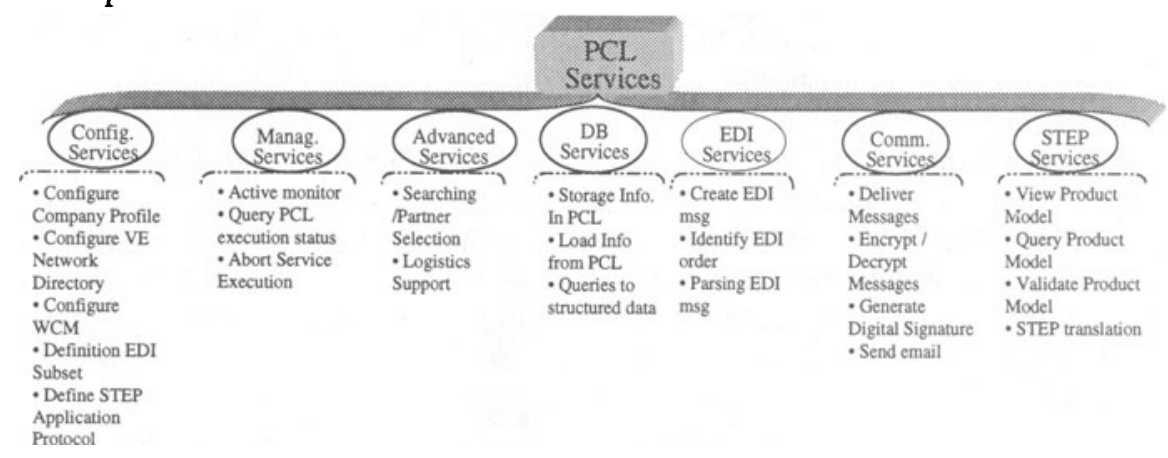

Figure 3 - Hierarchy of PCL services

PCL is offering a set of services allowing the participation of SMEs in a VE environment. Figure 3 shows the initial set of PCL services, grouped by functionalities, namely: configuration services, management services, advanced services, database services, EDI services, communication services, and STEP services. These services are supported by different software modules of the PRODNET platform. 
These services can be classified according to two perspectives: their purpose and their structure. According to the first perspective, there are two classes of PCL services: High-level and Support services.

The high-level class is composed by the advanced services, the configuration services, and some management services. The support level is composed by internal services provided by each PCL module, namely: EDI services, DIMS (Database) services, PCI services (communication services), STEP services and some lower level management services.

The second classification perspective considers the procedure adopted to request a service. There are two kinds of service: simple and composite services. Simple services can be directly invoked, through a human interface, by an operator, and performed as a single action. Composite services are usually requested through the so-called PCL workflow model (described in the next section), because they must be executed in several steps. Examples of simple services are: visualize a product model, send product model, STEP translation (from/to) native formats, store information in PCL, load information from PCL, request catalog of products, request quality information, or request shared information. Examples of composite services are: receive/send a client order, receive a product model, receive a products catalog request, receive a quality information request, receive a historical production $\log$ request, etc.

\subsection{Workflow concepts applied to PCL Management}

In order to provide a flexible way to configure the PCL behavior, it is convenient to separate the catalog of services from the control flow. The control flow should not be embedded in the PCL code but rather should be configurable to each company environment. In order to achieve this, PRODNET adopted the concepts developed by the Workflow Management Coalition Group - WfMC [WfMC94], namely the Workflow Reference Model [Camarinha97c] as the underlying framework.

A workflow model can be used to design fully or partially automated solutions for certain business process within an enterprise and across multiple enterprises [Miller96]. The Workflow Management Coalition (WfMC) is a non-profit, international organisation whose mission is to promote the use of workflow through the establishment of standards for software terminology, interoperability and connectivity between workflow products. In [Camarinha97c] several benefits of the adoption of this approach to PCL management were pointed out including:

- PCL behavior is supported by the PCL Workflow Model (PWM);

- PWM is written in a formal language (WPDL);

- LCM is implemented as a workflow engine.

PCL services are activated by messages coming from the VE environment and from the internal module of the company. Inside PCL, each message can pass through several modules. The "path" to be followed by any message is configured in the PWM. For instance, an EDI order coming through the PCI goes to LCM, then to DIMS (storage and identification), after that to EDI (EDI validation), and 
finally it goes to the PPC system. PWM is used by LCM to guide the execution of all actions related to the treatment of each message inside PCL.

\subsection{Multilevel Workflow concept}

This workflow approach can be generalized to a hierarchical coordination model. On one hand, there are two clearly distinct levels: the global VE coordination level and the coordination of the cooperation events inside each member enterprise. On the other hand, even inside a single VE member, there are a large number of cooperation events to be handled, which require the definition of several subworkflow plans. In order to facilitate the understanding and management of such plans the definition of a hierarchy of coordination levels seems adequate.

PRODNET adopted a three-level coordination approach corresponding to the following domains: basic PCL communications, company level coordination, and VE level coordination.

In the first domain, the PCL workflow engine (i.e., the Local Coordination Module, LCM) is implemented to support the customisation of the PCL behavior according to the internal rules of a company. LCM is a reactive, message-driven, engine. This means it acts based on external messages. In the second domain, the Company workflow engine (CWE) is implemented to support the monitoring and control of the accomplishment of the rights/obligations implied by the contracts between the company and its suppliers or clients. This engine is a "live" entity working in a loop, since the contract rules can be time-based (periodic and cyclic). The inter-operation between the two engines is required because all services offered by LCM might be used by CWE. In the third domain, the VE global workflow engine (VWE) is implemented to support the role of a VE coordinator, enabling a VE to work cooperatively as a single unit. This engine has a hybrid behavior, reactive and live. Actions at the VE level might occur on request or be time-based (for instance, execution of pre-programmed tasks in a VE global scheduling). In an analogous way, the interoperation between the three engines is required because VWE can use both CWE and LCM kernel as service providers (Figure 4).

LCM Workflow level. The activities included at this level are the ones described in section 3.2.

Company Workflow Level. The CWE is based on the rights and obligations defined in the contract that establishes the relationship between enterprises in the VE. The contract clauses have two main characteristics that need to be supported: they are cyclic and periodic. For instance, one clause may specify a long-term supply contract and requiring: "in the first week of each month both orders and orders forecast for the next month must be sent to the supplier"; another clause may say: "an order is considered accepted in 5 days after being sent, if the supplier has not sent any answer during this period".

A draft workflow model to support the first clause is presented in figure 5. "Contract Monitoring" is the main activity to monitor the whole contract. "Orders schedule" flow represents the first example clause. It is composed of three activities: periodic order, order forecast and send order. These activities use some 
services (timer and PPC services) and they might have interaction with the human operators. The activity "Periodic Order" has to be performed periodically (the first week of each month). Thus, it uses a "timer service" to wake up. The two other activities are then executed: "Order forecast" and "Send order". Both of them can request automatic services from PPC or send a message to the human operator. The flow proceeds to the temporized activity (label A1) and the loop is started again.

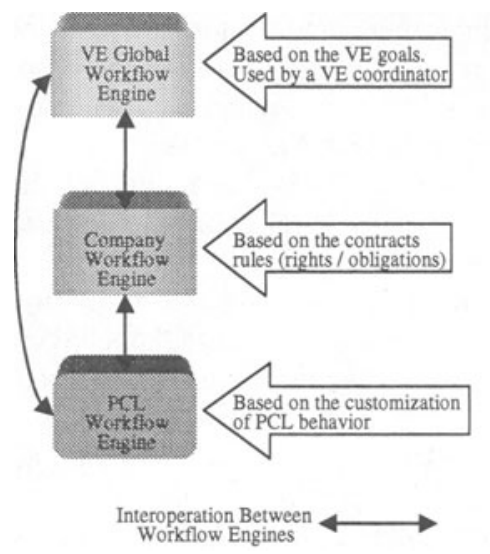

Figure 4 - Hierarchical Workflow Concept

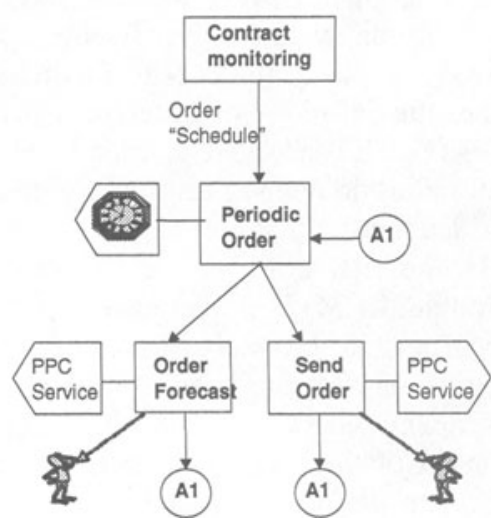

Figure 5 - Workflow model for a contract's clause

VE Workflow Level. This level is mainly related to the Advanced Coordination Functionalities (ACFs) and the initial approach is to create a VE workflow model to support the representation of the global VE goals. The GWE might work on this model, interacting with ACFs, CWE and PWE. This part is still in its initial development phase.

\subsection{Local Configuration Module (LCF)}

The LCF is basically a graphical editor which allows a particular PCL configuration, according to the specific needs of a company and the structure of the VEs in which that company is involved in. The LCF deals with various classes of information, namely: workflow models, company profile and a VE network directory. Three workflow models are used to support the implementation of the hierarchical workflow concept: the PCL workflow model (PWM), the company workflow model (CWM), and the VE global workflow model (GWM).

The LCF development has started with the Workflow Editor, which is aiming to provide a comfortable environment for workflow edition. Figure 6-a shows a small part of a PWM, dealing with the reception of EDI orders and STEP messages.

The graphical language used by the Workflow Editor to represent its entities follows the standardized symbols defined by the WfMC. The initial set used by LCF is:

Activity: represents the "control points" used to monitor message handling.

囚 Requested service: each of the services provided by PCL. 
$\rightarrow$ Transition: used to connect two activities. Conditions and validations can be associated to a transition.

O Participants: to define the person involved in/responsible for an activity.

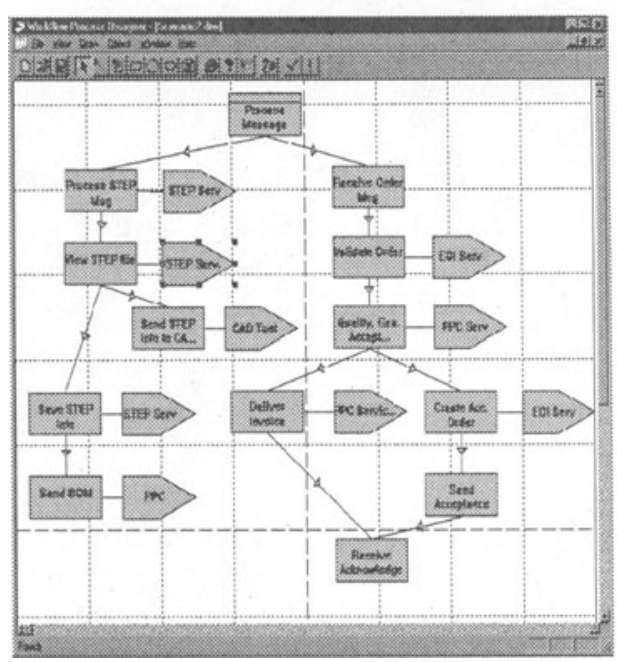

PCL WORKFLOW MODEL

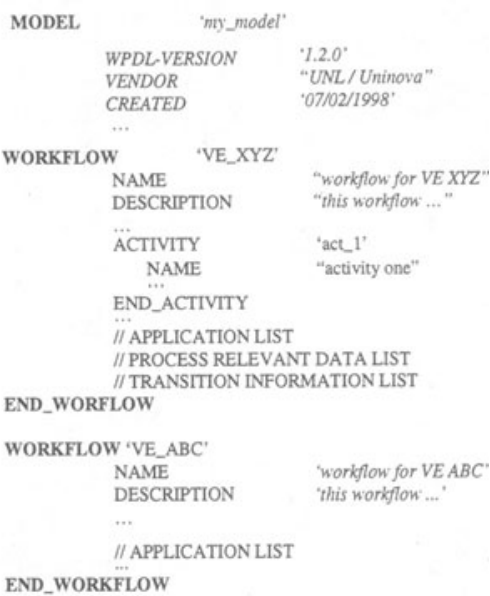

Figure 6 - a) Company Workflow Model editor

b) WPDL language example

The PWM is used also to support simultaneous participation of a company in different VEs [Camarinha97c]. The company has a set of data related to each VE it is involved in. Thus, PCL offers to a company two or more possible different ways to handle the same "business operation" within different VEs.

Figure 6-b) illustrates a workflow plan written in the WPDL language.

The VE Network Directory (VEND) editor is another module that allows the configuration of all information related to the VE itself, such as: VE coordinator profile, VE members' properties, relationships among VE partners, access rights, network information (IPs, email, ftp server, etc.), etc.

\subsection{Local Coordination Module (LCM)}

The LCM acts as the executor of the PCL. All messages/events that come to PCL, both from the internal legacy systems and from the external world, are handled and monitored by the LCM according to the PWM defined in the LCF. One important aspect is that LCM intends to perform only monitoring and control actions. The data manipulation is left to the PCL component called DIMS. LCM needs to know just an identifier which provides the non-ambiguous reference to the contents of a given message.

LCM is composed by two modules: the LCM Kernel and the LCM Monitor. The Kernel is the workflow engine itself which has to control the execution flow of all messages coming to PCL; the Monitor is the interface through which a human operator can inspect what is happening inside PCL and interact with the LCM kernel. For instance, the user can stop services execution, visualize the messages 
coming to the company, trace all services being performed by PCL, or deal with unexpected events found by LCM.

The LCM kernel is split in two modules: the Workflow Loader and the Workflow Executor (fig. 7).

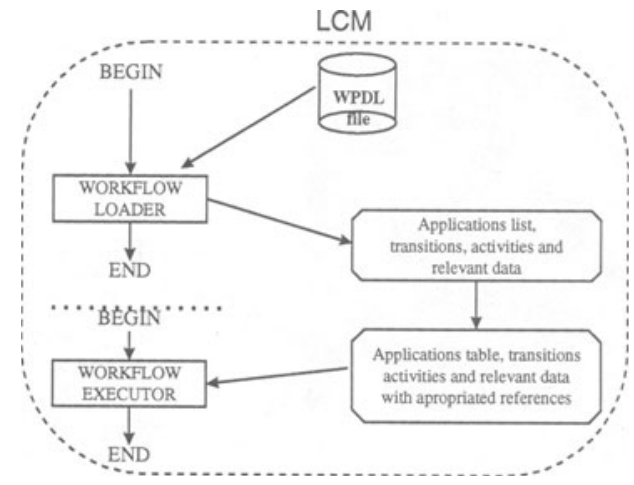

Figure 7 - LCM components

BEGIN and END represent activities performed internally by the LCM kernel.

The Workflow Loader is responsible for reading the ".wpdl" file and for preparing all the temporary information which will be used to instanciate the workflow executor.

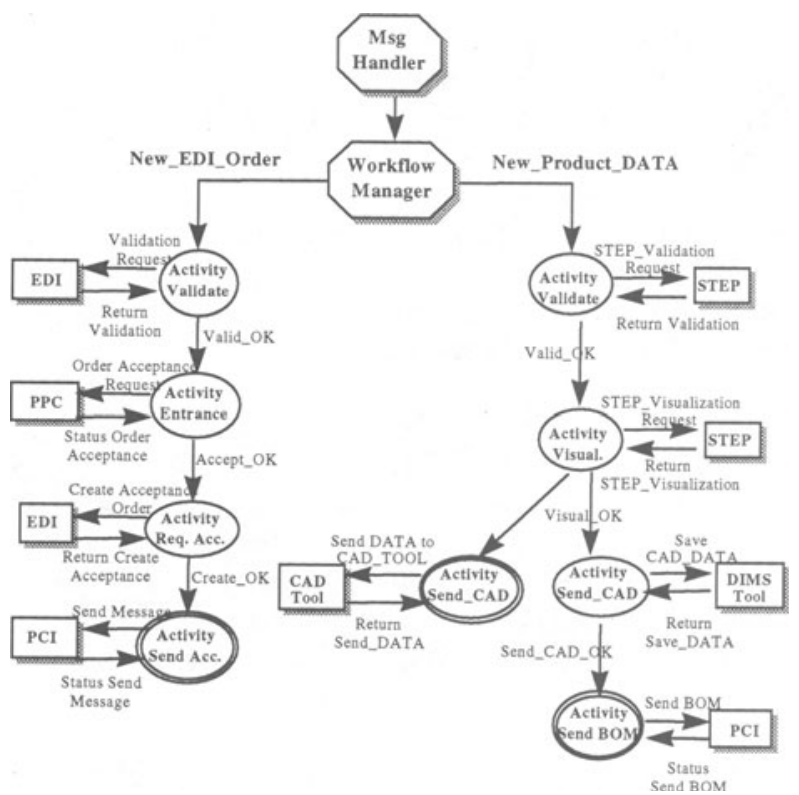

Figure 8 - A detailed example of worflow 
The Workflow Executor is the "real" controller and executor of the PWM. This module is, on its turn, composed by the Message Handler and the Workflow Manager. The first one is responsible for receiving and sending all messages to/from the LCM. It continuously checks if a message has arrived at its "mailbox". The manager is responsible for the execution of the workflow branches defined for each type of message received by PCL.

Figure 8 shows a detailed example of configuration and execution of two messages: an EDI order and a Product Data message. Each activity is related to a PCL service made available to the user. The execution flow only proceeds to the next activity if the requested service is performed with no errors.

\subsection{Relationship between LCF and LCM}

Figure 9 presents a general overview of the relationship between LCF and LCM. The LCF module receives the Company Workflow Model from a human operator and generates a file written in the WPDL format. This file is used by the LCM to build a Finite Automata which controls all the activities, transitions and the applications defined to deal with each message, for that particular company. The LCM kernel interacts with a human operator through the LCM monitor.

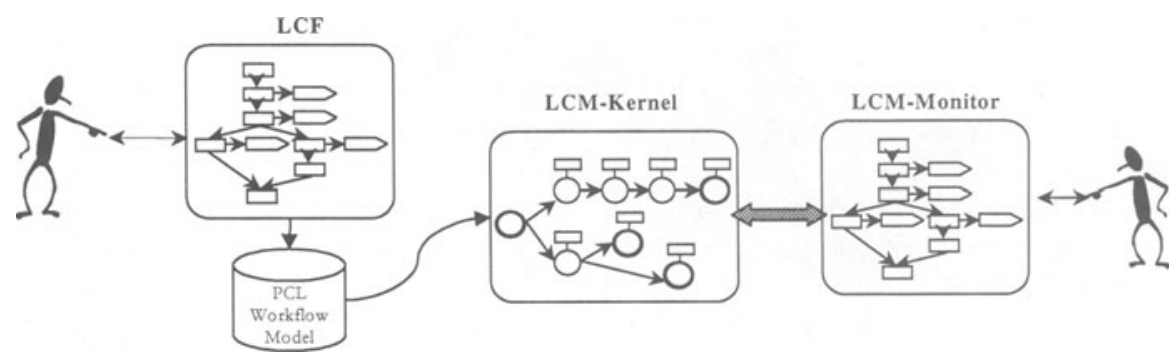

Figure 9 - Relationship between LCF, LCM and monitor

\subsection{The other workflow engines}

The other workflow levels are still being developed but the general principle is to use a similar approach as in the case of LCM.

\section{CONCLUSIONS AND OPEN QUESTION}

The configuration and coordination issues within a computational platform to support the VE environment were presented, based on the Esprit PRODNET II results. The supporting technology, as well as the future directions were also discussed. However, as PRODNET II is still in its early stages, several questions remain open and require further work. Examples:

-The list of services to support cooperation is not fully defined yet.

-The aspects related to the extension of the workflow concepts towards the VE global coordination are not fully identified. 
-The hierarchical model to support the workflow concepts is not completely tested.

Some extensions might be necessary.

-The implementation phase is progressing and a practical assessment is needed.

However, a preliminary validation based on the interaction with end users shows that the global approach being followed by PRODNET II is a promising one.

\section{Acknowledgments}

The authors would like to thanks the PRODNET II consortium, and the support from both the European Commission and the Brazilian governmental research funding agency (CNPq).

\section{REFERENCES}

Afsarmanesh, H.; Garita, C.; Hertzberger, L.O.; Santos Silva, V. - Management of distributed information in Virtual Enterprises. Proc. of ICE'97 $4^{\text {th }}$ Int. Conf. on Concurrent Engineering, Nottingham, October 8-10, 1997.

Afsarmanesh, H.; Camarinha-Matos' L.M. - Federated Information Management for Cooperative Virtual Organizations, Proceedings of DEXA'97 - Int. Conf. On Data Bases and Expert Systems Applications (L N in Computer Science, Springer-Verlag), Toulouse, Sep 97.

Camarinha-Matos, L.M.; Carelli, R.; Pellicer, J.; Martin, M. - Towards the virtual enterprise in the food industry, ISIP'97 - OE/IEEE/IFIP Int. Conf. on Integrated and Sustainable Industrial Production, Lisboa, May 1997.

Camarinha-Matos, L.M.; Afsarmanesh, H.; Garita, C.; Lima, C. - Towards an Architecture for Virtual Enterprises, The 2nd World Congress on Intelligent Manufacturing Processes \& Systems, Budapest, Hungary, June 10-13, 1997.

Camarinha-Matos, L.M.; Lima, C.; Osório, L. - The PRODNET platform for production planning and management in virtual enterprises. Proc. of ICE' $974^{\text {th }}$ Int. Conf. on Concurrent Engineering, Nottingham, October 8-10, 1997.

Miller, J.A.; Sheth, A. P.; Kochut, K.J.; Wang, X. - CORBA-Based Run-time Architectures for Workflow Management Systems. Journal of Database Management, Special Issue on Multidatabases, Vol. 7, No. 1, 1996, pp. 16-27.

Mizuta, H. - Virtual Enterprise Projects in Japan, Proc. of ICE' $974^{\text {th }}$ Int. Conf. on Concurrent Engineering, Nottingham, October 8-10, 1997.

Niiip96- The NIIIP Reference Architecture, 1996, http://www. niiip.org.

WfMC- Workflow Management Coalition (1994) - The Workflow Reference Model - Document Number TC00 - 1003, Issue 1.1, Brussels Nov 29, 1994

Zarli, A.; Poyet, P. et al. - Integrating Emerging IT paradigms for the Virtual Enterprise: the VEGA platform. Proc. of ICE' $974^{\text {th }}$ Int. Conf. on Concurrent Engineering, Nottingham, October 8-10, 1997. 